\title{
EVALUACIÓN E INTERVENCIÓN PSICOFISIOLÓGICA TÉRMICA DE SESIÓN ÚNICA DE ESTRÉS SOCIAL EN ESTUDIANTES DE PSICOLOGÍA
}

\author{
David Alberto Rodríguez-Medina, José Joel Vázquez Ortega y Griselda Alonso Hernández \\ Universidad Autónoma Metropolitana \\ México
}

\begin{abstract}
RESUMEN
El estrés social agudo induce un incremento de la actividad simpática, mientras que la relajación promueve actividad parasimpática. Sin embargo, algunas personas no logran regular su balance autonómico (estrés-relajación), por lo que se propone evaluar el efecto de una intervención psicofisiológica de sesión única. Participaron estudiantes de Psicología $(n=15)$, quienes completaron una evaluación psicométrica de medidas de distrés (ansiedad, depresión, estrés postraumático, alexitimia y nivel subjetivo de estrés) previa a la intervención. Después se aplicó el trier social stress test (TSST-Cog), un protocolo cognitivo para inducir estrés social agudo, seguido de una estrategia de relajación, imaginería guiada (IG). Mediante un diseño de medidas repetidas se registró su temperatura nasal y se comparó la diferencia térmica entre estrés y relajación. En seguida los estudiantes que reportaron un nivel de distrés en las medidas psicométricas se registraron en dos subgrupos para aplicar un procedimiento de condicionamiento térmico en manos (grupo de relajación y grupo control). Los resultados revelaron una asociación $(p<0.05)$ entre la temperatura nasal y las medidas de distrés; un decremento de la temperatura nasal post-TSST-Cog (acompañado de un aumento de percepción de estrés) y un incremento térmico (asociado a una disminución de estrés) post-IG con diferencia estadística entre ambos procedimientos $(p<0.01)$. Para los participantes con distrés, el procedimiento de condicionamiento térmico logró revertir el descenso de temperatura $(p=0.05)$, mientras que los estudiantes sin el procedimiento no tuvo cambios post-IG $(p=0.15)$. Se concluye que el uso del protocolo estrés-relajación se puede utilizar como procedimiento diagnóstico del funcionamiento emocional, así como el uso del condicionamiento térmico.
\end{abstract}

Palabras Clave:

TSST-Cog, temperatura-nasal, ansiedad, imaginería-guiada, condicionamiento-térmico.

\section{SINGLE-SESSION THERMAL PSYCHOPHYSIOLOGICAL EVALUATION AND INTERVENTION OF SOCIAL STRESS IN PSYCHOLOGY STUDENTS}

\begin{abstract}
Acute social stress induces an effect of sympathetic activity, while relaxation promotes parasympathetic activity. However, some people fail to regulate their autonomic balance (stress-relaxation), so that the effect of a single session psychophysiological intervention can be evaluated. Participate pre-grade psychology students $(n=15)$ who completed a psychometric evaluation of distress measures (anxiety, depression, post-traumatic stress, alexithymia and subjective perception of stress). Subsequently, the Trier Social Stress Test (TSST-Cog) was applied, a behavioral protocol to induce acute social stress, the follow-up of guided imagery (GI) search strategy. Through a repeated measures design, it is about its nasal temperature and the thermal difference between stress and relaxation is compared. Subsequently, the students who reported a level of distraction in the psychometric measures were randomized into two subgroups to apply a procedure of thermal conditioning on the hands - relaxation and a control group. The results are revealed in an association ( $p<0.05$ ) between the nasal temperature and the measures of distress; a decrease in the nasal temperature during the TSST-Cog (accompanied by an increase in the perception of stress) and a thermal increase $(p<0.01)$. For the participants with the thermal conditioning procedure, the decrease in temperature was reversed $(p=0.05)$, while the students without the procedure had no post-GI changes $(p=0.15)$. It is concluded that the use of the protocol. TSST-Relaxation can be used as a diagnostic procedure of emotional functioning, as well as the promotion of thermal conditioning in those who do not benefit from the procedures of traditional practice.
\end{abstract}

Keywords:

TSST-Cog, nasal temperature, guided imagery, thermal-conditioning.

Bitácora del Artículo:

| Recibido: 22 de Julio de 2019 | Aceptado: 31 de Julio de 2019 | Publicado en línea: Julio - Diciembre 2020 | 


\title{
EVALUACIÓN E INTERVENCIÓN PSICOFISIOLÓGICA TÉRMICA DE SESIÓN ÚNICA DE ESTRÉS SOCIAL EN ESTUDIANTES DE PSICOLOGÍA
}

\author{
David Alberto Rodríguez-Medina, José Joel Vázquez Ortega y Griselda Alonso Hernández \\ Universidad Autónoma Metropolitana \\ México
}

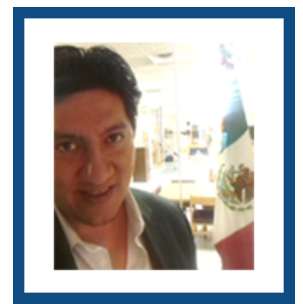

David Alberto Rodríguez Medina

UAM-Iztapalapa

Correo: drodriguezm@xanum.uam.mx

Doctor en Psicología y Salud por la UNAM, miembro de la Sociedad Interamericana de Psicología, profesor investigador visitante titular B de tiempo completo en la Universidad Autónoma Metropolitana, unidad Iztapalapa. Investigador Nacional Nivel I del SNI, CONACYT.

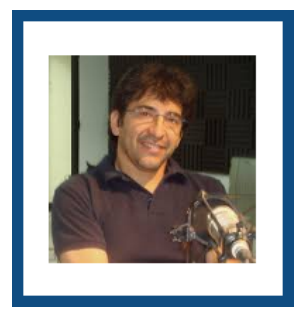

José Joel Vázquez Ortega

UAM-Iztapalapa

Correo:

Profesor investigador titular $\mathrm{C}$ de tiempo completo, candidato a doctor. Premio Mexicano de Psicología 2011. Coordinador y coautor de varios libros de Psicología Social, así como diversos artículos publicados en distintos países internacionales. Jefe del área de investigación en Procesos Psicosociales de los Fenómenos Colectivos de la Universidad Autónoma Metropolitana, unidad Iztapalapa.

\section{CONTRIBUCIÓN DE LOS AUTORES}

David Alberto Rodríguez-Medina: Concepción del estudio, diseño de investigación, análisis de datos y redacción del artículo. | José Joel Vázquez Ortega: Planeación y organización del estudio | Griselda Alonso Hernández: Aplicación del procedimiento e instrumentación para la recolección de datos..

\section{AGRADECIMIENTOS}

Apreciamos el apoyo invaluable de Gabriela Jael Pérez García, así como a nuestros colegas del área de Investigación en Procesos Psicosociales de los Fenómenos Colectivos de la UAM-I: Omar Manjarrez, Mario Carranza, Marisol Pérez y José Gutiérrez-Fiallo. También al doctor Benjamín Domínguez y colegas del grupo Mente-Cuerpo de la Facultad de Psicología, UNAM (Mariana, Sandra, Nadia, Mayra, Li, Carolina, Sara, Omar, Esael y Erick).

\section{Datos de Filiación de los Autores}

Universidad Autónoma Metropolitana, Unidad Iztapalapa

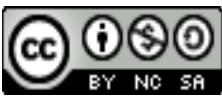

Copyright: (c) 2020 Rodríguez-Medina, D.A., Vázquez-Ortega, J.J. \& Alonso-Hernández, G.

Este es un artículo de acceso abierto distribuido bajo los términos de la licencia Creative Commons Reconocimiento-NoComercial 4.0 Internacional, por lo que su contenido gráfico y escrito se puede compartir, copiar y redistribuir total o parcialmente sin necesidad de permiso expreso de sus autores con la única condición de que no se puede usar con fines directamente comerciales y los términos legales de cualquier trabajo derivado deben ser los mismos que se expresan en la presente declaración. La única condición es que se cite la fuente con referencia a la Revista Digital Internacional de Psicología y Ciencia Social y a sus autores. 


\section{TABLA DE CONTENIDO}

INTRODUCCIÓN

MÉTODO

Participantes, 343

Materiales, 344

Procedimiento, 344

Mediciones, 344

Análisis estadísticos, 345

\section{RESULTADOS}

1) Evaluación psicométrica, 345

2) Asociaciones entre la temperatura nasal y las medidas psicosociales, 345

3) Evaluación psicofisiológica no invasiva y colectiva de estrésrelajación, 345

4) Efecto de la estimulación térmica en manos respecto a la temperatura nasal, 346

\section{Discusión}

CONCLUSIONES 


\section{INTRODUCCIÓN}

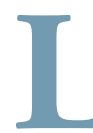

os procedimientos de evaluación psicofisiológica de estrés, referente a la actividad autonómica (respuesta cardiovascular, temperatura periférica o galvánica de la piel) o la activación somática (muscular, palpebral o respiratoria), tradicionalmente utilizan el registro mediante sensores adheridos a la piel. De manera reciente se han incorporado medidas no invasivas, como la recolección salival de cortisol (actividad endocrinológica) y/o la muestra de citoquinas proinflamatorias (actividad inmunológica) (Allen, Kennedy, Cryan, Dinan y Clarke, 2014; Rodríguez-Medina et al., 2019).

Sin embargo, el costo de estas medidas psicofisiológicas tradicionales es elevado; requiere un entrenamiento profesional para la instrumentación y manejo de equipo, así como un tiempo considerable de evaluación, porque en general los protocolos de evaluación se hacen de manera individual y son escasos los estudios grupales con medidas psicofisiológicas (Von Dawans, Kirschbaum y Heinrichs, 2011).

Una opción de registro psicofisiológico no invasiva es el uso de la imagen térmica infrarroja, la cual es una herramienta que permite valorar en tiempo real la actividad autonómica periférica (rostro y manos) sin necesidad de tocar al participante durante el desarrolo de alguna tarea afectiva o cognitiva (Cardone y Merla, 2017).

El uso potencial de un sensor de temperatura infrarrojo se ha descrito en diversos protocolos de evaluación autonómica (Cardone y Merla, 2017; Rodríguez-Medina y Domínguez-Trejo, 2018) como una medida psicofisiológica útil que permite ponderar el balance simpático/parasimpático. Dicho balance neurofisiológico y autonómico lo explica la teoría Polivagal: lucha/huida ante un agente estresor; o el involucramiento social ante una situación de confianza. Esta capacidad de transición entre un estado con predominio simpático a uno parasimpático permite ponderar los recursos afectivos de una persona (Porges, 2003).

Una tarea estandarizada para evaluar los efectos psicofisiológicos del estrés social agudo es el Trier Social Stress Test (TSST), cuyos efectos psicofisiológicos se han documentado respecto al sistema nervioso simpático (actividad cardiovascular), endocrinológico (cortisol, alfa amilasa) e inmunológico (citoquinas inflamatorias) (Rodríguez-Medina, Domínguez, Leija, Ortíz y Carreño, 2018). Ante la presentación del estrés social agudo los vasos sanguíneos se constriñen e inducen un enfriamiento en manos (Cruz-Al- barran et al., 2018) y nariz (Rodríguez-Medina et al., 2018). En contraparte, las estrategias de relajación disminuyen la actividad simpática promoviendo la respuesta parasimpática, cuyo resultado es el aumento en la temperatura periférica (Rodríguez-Medina et al., 2018).

Sin embargo no todas las personas se benefician de los procedimientos psicológicos de relajación. Es decir, no todas las personas logran aumentar su temperatura periférica, sobre todo si tienen un historial de sucesos traumáticos afectivos y/o si su actividad inflamatoria es elevada (Lasselin et al., 2016).

Cuando la persona no logra incrementar la temperatura por sí misma, se ha sugerido el uso de fuentes de calor externo para facilitar la respuesta parasimpática (Vergara, et al. 2018); basados en el estudio de Raison, Hale, Williams, Wager y Lowry (2015), quienes describieron las vías en termosensoriales periféricas y su procesamiento neurofisiológico central, que influye en la respuesta afectiva, una temperatura periférica cálida promueve la sensación de agrado y viceversa.

Los objetivos del presente estudio fueron: 1) analizar el comportamiento de la temperatura periférica nasal ante la presentación de un protocolo línea base-estrés-relajación administrado de manera colectiva en estudiantes de pregrado en Psicología, y evaluar las diferencias estrés-relajación en la temperatura nasal; 2) explorar el efecto de la estimulación térmica en estudiantes con sintomatología de distrés psicológico en la temperatura nasal y contrastar el efecto de la intervención sin la estimulación térmica de la misma medida; 3 ) examinar si la temperatura nasal (como medida del balance autonómico) tiene relación con el autorreporte de los puntajes de escalas psicométricas asociadas a estrés (ansiedad, depresión, sintomatología de estrés postraumático, alexitimia) en los estudiantes de Psicología.

\section{MÉTodo}

\section{Participantes}

Mediante una convocatoria para la capacitación de los estudiantes de servicio social se utilizó un muestreo no probabilístico, intencional por conveniencia; participaron de manera voluntaria 15 estudiantes universitarios de pregrado en Psicología, con edad media $=20.714( \pm$ 0.994) años. Se consideraron los criterios de inclusión para participar en el estudio: ayuno de al menos dos horas, libres de cosméticos faciales. Como criterio de exclusión se consideró que no podían participar los estudiantes con fiebre. Los estudiantes que quisieran retirarse del estudio podían hacerlo en cualquier momento. 
Todos los participantes dieron su consentimiento informado con base en la declaración de Helsinki.

\section{Materiales}

Non contact infrared body Thermometer DM300巴 (DM300®, 2016). Termómetro de luz infrarroja con diámetro de $0.5 \mathrm{~cm} 2$, el cual registra la temperatura de la piel entre los $0{ }^{\circ} \mathrm{C}$ a $100^{\circ} \mathrm{C}$. Se registró la temperatura de la punta de la nariz.

Bolsa de gel térmico Home Care $\AA$. Medidas: $10 \times 9$ $\mathrm{cm}$ que al activarse logra una temperatura de casi $41{ }^{\circ} \mathrm{C}$ durante un periodo de entre 15 a 20 minutos.

Patient Health Questionarie (PHQ-4) (Kroenke, Spitzer, Williams y Löwe, 2009). Es un cuestionario de autoaplicación de cuatro reactivos (para evaluar ansiedad y dos para depresión) con escala tipo Likert de 0 a 3, según la frecuencia de los síntomas. Se consideran los siguientes niveles de distrés: puntaje entre 0 y 2 = sin distrés; puntuación entre 3 y 5 = nivel bajo; puntuación entre 6 y 8 $=$ nivel moderado; $y$ puntaje entre 9 y $12=$ nivel alto.

Primary Care of Post-Traumatic Stress Disorder (Prins et al., 2003). Instrumento autoaplicable de sintomatología de trastorno de estrés postraumático del DSM-IV-R, con cuatro preguntas con opción de respuesta dicotómica (sí) no). Una puntuación $3 \geq$ indica presencia de TEP.

Escala de alexitimia de Toronto-10. Instrumento de autorreporte de alexitimia (dificultad para identificar y expresar emociones y sentimientos hacia sí mismo y hacia los demás). Validado en población estudiantil universitaria mexicana (Durán, 2007), tiene cinco niveles de alexitimia, que van desde nula (0-5) hasta completa $(32 \geq)$.

Trier Social Stress Test - Cognitivo (TSST-Cog). Basado en el Trier Social Stress Test (Kirschbaum, Pirke y Hellhammer, 1993), prueba conductual de laboratorio para inducir estrés agudo; se adaptó una versión breve cognitiva (Rodríguez-Medina et al., 2018). A todos los participantes se les proporcionó una hoja con las indicaciones de preparar mentalmente un discurso y una tarea aritmética para después pasar al frente del grupo a exponer ambas tareas, con videocámara y cámara térmica infrarroja encendida (desde luego no pasaron a efectuar la tarea, pues se buscaba evaluar el efecto cognitivo de la evaluación social).

Puntaje de estrés. Nivel de estrés subjetivo en una cuadrícula horizontal que va del 0 ("Poco o nada") al 10 ("Máximo estrés").

Relajación. De manera grupal se indujo un ejercicio de imaginería guiada.

\section{Procedimiento}

Como parte de sus tareas de capacitación continua, los estudiantes de servicio social asistieron a la convocatoria para participar en el estudio en una sala iluminada, ventilada, a temperatura controlada de $22^{\circ} \mathrm{C}$, con sillas y mesas adyacentes. Se reunieron entre las 09:00-10:00 horas en una sala a temperatura ambiente controlada a $22{ }^{\circ} \mathrm{C}$. Se les explicó el objetivo del estudio y dieron su consentimiento. Ya distribuidos en la sala se les aplicó la evaluación psicométrica mediante la administración de cada escala hasta completar su evaluación por autorreporte. Luego de permanecer sentados en la sala al menos 10 minutos para aclimatarse a la temperatura ambiente, se les solicitó que permanecieran tres minutos quietos en sus asientos (línea base), y al término de es periodo se registró su temperatura nasal y se les pidió que calificaran su nivel subjetivo de estrés. En seguida se les administró el TSST-Cog (tres minutos) mientras se colocaba una silla y dos cámaras (una de video y una térmica) al centro de la sala para hacerles creer que pasarían a desarrollar las indicaciones del TSST-Cog. Transcurridos los tres minutos se volvió a registrar su temperatura nasal y se les solicitó de nuevo calificar su nivel subjetivo de estrés. Al final se implementó la estrategia de relajación mediante un ejercicio de imaginería guiada durante los siguientes tres minutos para luego volver a registrar su temperatura nasal y su nivel de estrés subjetivo. Además, en los estudiantes que tuvieron una puntuación clínica de distrés y que no lograron revertir el efecto térmico del TSST, se implementó una intervención mediante el condicionamiento de la respuesta autonómica térmica sosteniendo la bolsa de gel térmico (Home Care ${ }^{\circ}$, que al activarse alcanza una temperatura de casi $41^{\circ} \mathrm{C}$ ) en las manos mientras se les inducía el ejercicio de imaginería guiada al finalizar el ejercicio inicial.

\section{Mediciones}

Diseño de investigación prospectivo, transversal para la evaluación psicométrica, y diseño cuasiexperimental de medidas repetidas para el registro psicofisiológico de temperatura nasal e índice de estrés subjetivo (figura 1).

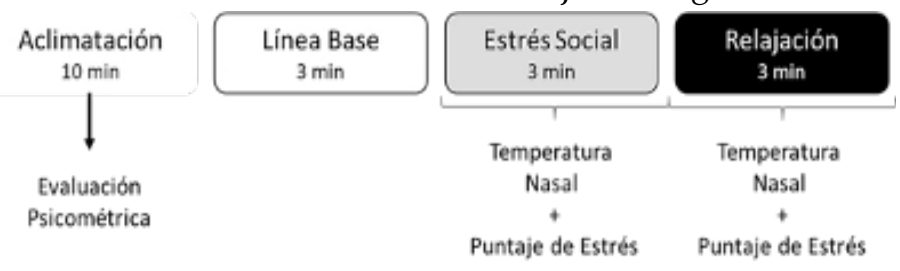

Figura 1. Diseño de investigación. Protocolo línea base-estrés-relajación y medidas de distrés. 
Artículo Empíricol Evaluación e Intervención... Rodríguez-Medina, Vázquez-Ortega QL Alonso-Hernández

\section{Análisis estadísticos}

Se extrajeron los datos descriptivos de las medidas psicométricas y psicofisiológicas explorando la normalidad de los datos con la prueba Kolmogórov-Smirnov ( $p<$ 0.05). Con base en la normalidad de cada variable se analizaron las relaciones entre ambas dimensiones de estrés y se obtuvieron coeficientes de correlación rho de Spearman y las regresiones lineales.

Para evaluar el efecto del protocolo de evaluación de estrés social y la relajación en el índice de estrés subjetivo y la temperatura nasal se hizo un análisis de medidas repetidas no paramétrico de Friedman. Además se examinaron las diferencias (incrementos/decrementos) de temperatura nasal $\left(\Delta \mathrm{T}{ }^{\circ} \mathrm{C}\right)$ y el índice de estrés ( $\Delta \mathrm{T}$ puntaje) entre: 1$)$ línea base-estrés; 2 ) estrés-relajación, y 3) se evaluaron con una prueba t de Student para muestras relacionadas.

Al final se evaluó el efecto de la estimulación térmica en manos respecto a la temperatura nasal durante el protocolo de evaluación psicofisiológica en los estudiantes con distrés (puntuación moderada o alta en las escalas psicosociales PHQ-4 o PC-PTSD) con la prueba estadística de Friedman.

En los análisis se consideró una significancia $\alpha=0.05$.

\section{Resultados}

\section{1) Evaluación psicométrica}

De la evaluación psicométrica se encontró que 33\% de la muestra presentaba un índice clínico de ansiedad de moderada a severa. De ellos, una persona presentó sintomatología de estrés postraumático y alexitimia, mientras que otro participante reportó un nivel moderado de ansiedad. En la tabla 1 se muestran los valores de las características psicosociales del grupo de estudiantes de Psicología.

Tabla 1.

Características psicosociales de los estudiantes de Psicología $(N=15)$.

\begin{tabular}{lll}
\multicolumn{1}{c}{ MedidA } & $\begin{array}{c}\text { Media (DESVIACIón } \\
\text { ESTÁNDAR) }\end{array}$ & $\begin{array}{c}\text { INTERVALO DE } \\
\text { CONFIANZA }\end{array}$ \\
\hline Ansiedad & $2.2(1.656)$ & {$[1.2829-3.1171]$} \\
\hline $\begin{array}{l}\text { Depresión } \\
\text { Trastorno de estrés } \\
\text { postraumático }\end{array}$ & $0.73(1.207)$ & {$[0.5315-1.8685]$} \\
\hline Alexitimia & $15.2(6.338)$ & {$[11.690-18.709]$} \\
\hline
\end{tabular}

\section{2) Asociaciones entre la temperatura nasal y las medidas psicosociales}

Se encontraron 12 correlaciones estadísticamente significativas $(p<0.05)$ entre la temperatura nasal, el índice de estrés basal o inicial previo a la evaluación al TSST-Cog, y entre las medidas psicosociales de distrés. La temperatura nasal se asoció de manera inversa con el índice de estrés, ansiedad y depresión; mientras las escalas psicosociales se asociaron de manera directa entre sí (figura 2).
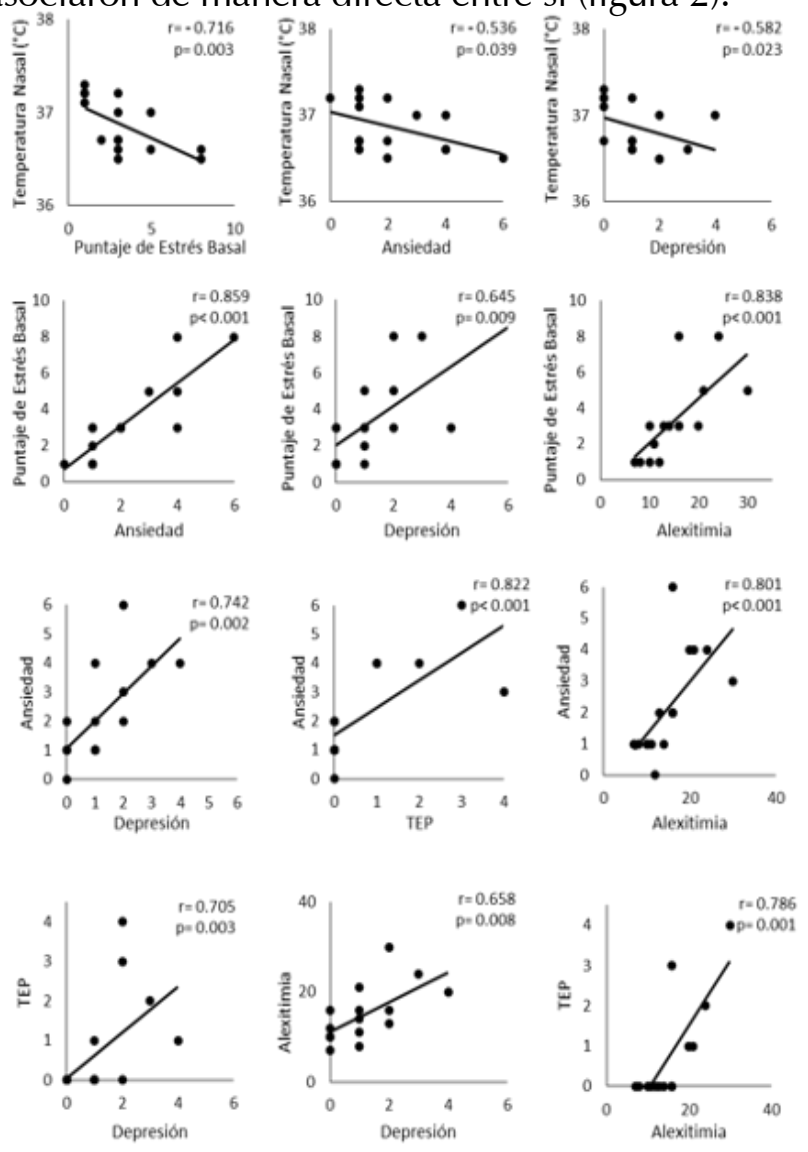

Figura 2.

Asociaciones entre la temperatura nasal y los puntajes psicométricos de distrés.

\section{3) Evaluación psicofisiológica no invasiva y colectiva de estrés-relajación}

Los análisis de medidas repetidas indicaron un efecto principal del protocolo del TSST-Cog y la relajación, estadísticamente significativo en el índice de estrés $\left(X^{2}=\right.$ $20.111(2), p=0.00004)$ y en la temperatura nasal $\left(X^{2}=\right.$ $22.945(2), p=0.00001$ ).

Para examinar los efectos específicos del TSST-Cog y la relajación se extrajeron los cambios entre las condiciones: 1) línea base-estrés, y 2) estrés-relajación. Los resultados mostraron una diferencia significativa entre 
TSST-Cog y la relajación respecto al índice de estrés $(Z=$ -3.417, $p=0.001)$, y a la temperatura nasal $(Z=-2.734$, $\mathrm{p}=0.006)$ (figura 3 ).

\section{a}
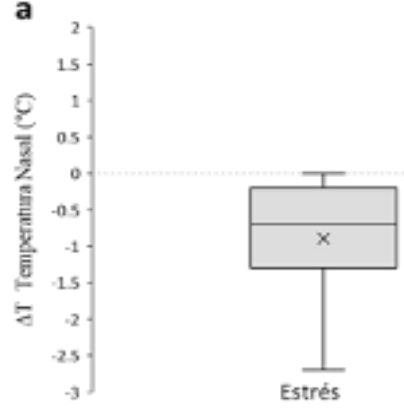

b

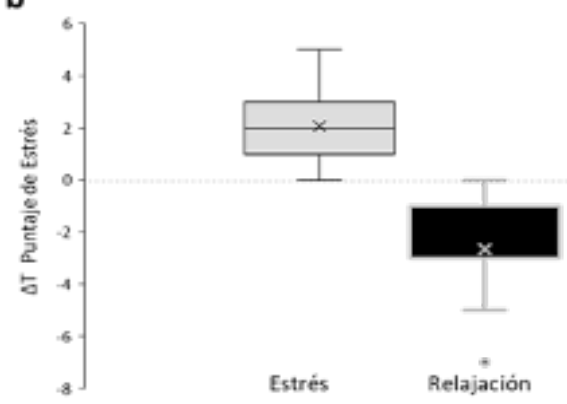

$p=0.005$

$p=0.001$
Figura 3.

Diferencias de temperatura nasal e índice entre estrés-relajación y su nivel.

\section{4) Efecto de la estimulación térmica en manos respecto a la temperatura nasal}

Entre los participantes que obtuvieron una puntuación clínica de ansiedad ( $N=5$ ) se aleatorizaron para evaluar el efecto de la aplicación $(n=3)$ o la no aplicación $(n=2)$ de estimulación térmica en manos respecto a la temperatura nasal durante el protocolo TSST-Cog y la relajación. Los resultados sugieren que la estimulación térmica en manos facilitó el incremento de la temperatura nasal durante la relajación $\left(X^{2}=6,(2), p=0.05\right)$, mientras que en el subgrupo con distrés sin estimulación térmica no logró la recuperación térmica nasal en la misma fase $\left(X^{2}=3.714,(2), p=0.156\right)$ (figura 4).

\section{Discusión}

Con base en los objetivos planteados en la presente investigación se logró evaluar la temperatura periférica nasal de manera colectiva durante el protocolo de TSST-Cog y la relajación. Esto sugiere una opción de recolección de datos psicofisiológicos en poco tiempo del sistema nervioso autónomo mediante el sensor de temperatura infrarrojo de manera no invasiva. Durante el estrés la temperatura nasal descendió, mientras que en la fase de relajación se incrementó respecto a la fase de estrés.

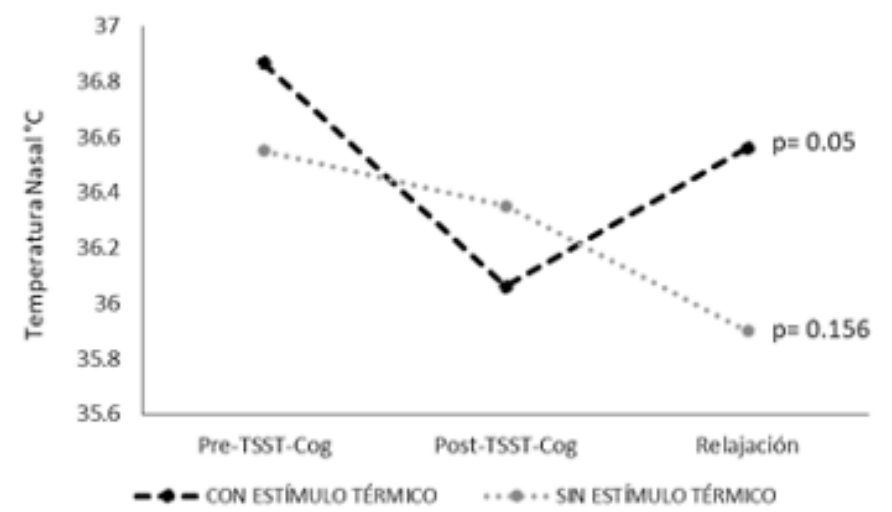

Figura 4.

Efecto de la estimulación térmica en manos sobre la temperatura nasal durante el protocolo estrés-relajación en participantes con ansiedad.

Otro objetivo propuesto fue examinar el efecto de la estimulación térmica en los participantes con puntuaciones clínicas de distrés. Para ellos, la estimulación térmica en manos facilitó su recuperación autonómica, mientras que aquellos sin estimulación no se recuperaron durante el periodo de relajación.

Como tercer y último objetivo planteado se encontró una asociación entre la temperatura nasal y las medidas de distrés psicológico (índice de estrés, ansiedad y depresión). También se reportan las asociaciones entre las medidas de distrés y la alexitimia, la cual se ha documentado su influencia en la caracterización de diversos trastornos psicosomáticos.

Entre las limitaciones del estudio se encuentra el tamaño de la muestra. Si bien la naturaleza del estudio es exploratoria, no se pretende generalizar los resultados sino describir la utilidad de la evaluación psicofisiológica no invasiva de manera colectiva mediante un sensor de temperatura infrarrojo y la influencia de los aspectos psicosociales en la respuesta al estrés en esta población. Los resultados presentados en este estudio coinciden con investigaciones antecedentes de los cambios en la temperatura periférica durante una tarea estresante (Rodríguez-Medina et al., 2018) y la relajación, así como la promoción de la actividad parasimpática mediante la estimulación térmica no nociceptiva (Rodríguez-Medina et al., 2018).

De acuerdo con Porges (2003), esta capacidad de transitar entre el predominio simpático al predominio parasimpático permitiría obtener un indicador de regulación autonómico en relación con el comportamiento afectivo, el cual facilitará conductas sociales de involucramiento 
social (en estado parasimpático), de lucha/huida con otro (estado simpático) o de distanciamiento social. Esta prueba estrés-relajación permite ponderar los recursos personales de activación e inhibición autonómica.

Para futuras investigaciones es necesario considerar aspectos metodológicos rigurosos como el tamaño y tipo de muestreo. Sería conveniente comparar poblaciones homogéneas considerables en el caso de participantes con distrés, e incluso valorar la influencia del ciclo menstrual de la temperatura periférica durante el desarrollo del TSST-Cog y la relajación. Esto permitiría obtener conclusiones potenciales más amplias del condicionamiento de la estimulación y las estrategias de relajación.

\section{Conclusiones}

El TSST-Cog induce un incremento en la actividad simpática promoviendo un decremento en la temperatura nasal, mientras la relajación promueve un aumento en la temperatura periférica disminuyendo el nivel el estrés.

El manejo del estrés social agudo podría considerarse una habilidad de regulación emocional sociocognitiva. Esto es de especial relevancia práctica para el pronóstico clínico: establecer un procedimiento diagnóstico del funcionamiento emocional para distinguir a quienes les funciona una estrategia de relajación inducida y a quienes requieren mayor apoyo psicológico (o incluso, psiquiátrico). En el caso de los estudiantes de Psicología que van a manejar grupos o casos particulares se sugiere abordar los aspectos psicosociales que impiden su desactivación simpática.

El involucramiento social promueve un estado de activación parasimpática (Porges, 2003), el cual se puede medir mediante el incremento de temperatura periférica, mientras que las personas con dificultades en la regulación de actividad autonómica pueden presentar un predominio de actividad simpática incluso al término del estresor y durante el periodo de relajación, lo cual los hace vulnerables a manifestar una elevada concentración de actividad inflamatoria, que a su vez influye en el estado anímico. Por supuesto es necesaria mayor investigación en el área de Psicofisiología Social para establecer normas térmicas en función del sexo, edad, índice de masa corporal; así como estudiar los índices y latencias de cambio térmico en diferentes regiones de interés observables de temperatura periférica.

La estimulación térmica artificial que se propuso en los estudiantes con distrés psicológico supone un procedimiento de condicionamiento autónomo de la respuesta parasimpática de vasodilatación: aplicación de calor en manos apareado con una estrategia psicológica de relajación post-estrés social agudo evaluado en la temperatura nasal (región de interés no estimulada directamente por el calor). Es necesario considerar con cautela los datos de este estudio exploratorio para efectuar réplicas en esta y otras poblaciones (sobre todo en población clínica/hospitalaria).

\section{Referencias}

Allen, A., Kennedy, P., Cryan, J., Dinan, T., \& Clarke, G. (2014). Biological and psychological markers of stress in humans: Focus on the Trier Social Stress Test. Neuroscience \& Biobehavioral Reviews, 38, 94-124. doi: 10.1016/j. neubiorev.2013.11.005

Cardone, D., \& Merla, A. (2017). New Frontiers for Applications of Thermal Infrared Imaging Devices: Computational Psychopshysiology in the Neurosciences. Sensors, 17(5), 1042. doi: $10.3390 /$ s17051042

Cruz-Albarran, I., Benitez-Rangel, J., Osornio-Rios, R., DominguezTrejo, B., Rodriguez-Medina, D., \& Morales-Hernandez, L. (2018). A methodology based on infrared thermography for the study of stress in hands of young people during the Trier Social Stress Test. Infrared Physics \& Technology, 93, 116-123. doi: 10.1016/j.infrared.2018.07.017

DM300®,N.(2016). Recuperadoel4deoctubrede2019de: https:// d3m9l0v76dty0.cloudfront.net/system/photos/2705008/ original/091a2827f12a4c29e09d06e0244fe9ea. pdf?1521030777

Durán, W. (2007). Validación de la Escala de Alexitimia de Toronto (TAS - 20). Con acceso el 22 de julio de 2019. http://catarina.udlap.mx/u dl_a/tales/documentos/lps/ weisel $\mathrm{d} \mathrm{m}$ / indice.html

Kirschbaum, C., Pirke, K., \& Hellhammer, D. (1993). The 'Trier Social Stress Test' - A Tool for Investigating Psychobiological Stress Responses in a Laboratory Setting. Neuropsychobiology, 28(1-2), 76-81. doi: 10.1159/000119004

Kroenke, K., Spitzer, R., Williams, J., \& Löwe, B. (2009). An UltraBrief Screening Scale for Anxiety and Depression: The PHQ-4. Psychosomatics, 50(6), 613-621. doi: 10.1016/ s0033-3182(09)70864-3

Lasselin, J., Kemani, M., Kanstrup, M., Olsson, G., Axelsson, J., \& Andreasson, A. et al. (2016). Low-grade inflammation may moderate the effect of behavioral treatment for chronic pain in adults. Journal of Behavioral Medicine, 39(5), 916924. doi:10.1007/s10865-016-9769-z

Prins, A., Ouimette, P., Kimerling, R., Camerond, R., Hugelshofer, D., \& Shaw-Hegwer, J. et al. (2003). The primary care PTSD screen (PC-PTSD): Development and operating characteristics. Primary Care Psychiatry, 9(1), 9-14. doi: 10.1185/135525703125002360

Porges, S. (2003). The Polyvagal Theory: phylogenetic contributions to social behavior. Physiology \& Behavior, 79(3), 503-513. doi: 10.22201/fpsi.20074719e.2018.2.293

Raison, C., Hale, M., Williams, L., Wager, T., \& Lowry, C. (2015). Somatic influences on subjective well-being and affective disorders: the convergence of thermosensory and central 
serotonergic systems. Frontiers in Psychology, 5, 1-22. doi: 10.3389/fpsyg.2014.01580.

Rodríguez-Medina, D. A., \& Domínguez-Trejo, B. (2018). La evaluación psicofisiológica con imagen térmica infrarroja en los procesos psicológicos. Revista Digital Internacional De Psicología Y Ciencia Social, 3(2), 227-241. doi: 10.22402/j. rdipycs.unam.3.2.2017.140.227-241

Rodríguez-Medina, D., Domínguez-Trejo, B., Ortiz-Omaña, M., Leija-Alva, G., \& Chavarría-Santiago, O. (2018). Efecto de la valencia afectiva del pensamiento sobre la temperatura nasal: Imaginería guiada y estrés psicosocial. Psicología $Y$ Salud, 28(2), 187-194. doi: 10.25009/pys.v28i2.2555

Rodríguez-Medina, D., Domínguez, B., Leija, G., Ortíz, M. \& Carreño, C. (2018). Efectos psicofisiológicos de la respiración diafragmática y la estimulación térmica sobre la actividad autonómica del estrés agudo. Acta De Investigación Psicológica, 8(2), 101-113. doi: 10.22201/ fpsi.20074719e.2018.2.293ç
Rodríguez-Medina, D., Leija-Alva, G., Domínguez-Trejo, B., Hernández-Pozo, M., Cruz-Albarrán, I., MoralesHernández, L., \& Marmolejo-Ramos, F. (2019). Effects of the Trier Social Stress Test on the distributions of IL-6 and MAP levels. Heliyon, 5(4), e01580. doi: 10.1016/j. heliyon.2019.e01580

Vergara-Aguirre, S. N., Rodríguez-Medina, D. A., Domínguez Trejo, B., Erandi-Tepepa, L., Vergara-Erdosay, A., Guidone Mariotti, N., \& Hernández-Santos, J. R. (2018). Estimulación térmica y analgesia hipnótica para la regulación autonómica e inflamatoria en pacientes con dolor crónico. Revista Digital Internacional De Psicología Y Ciencia Social, 4(2), 195-213. doi: 10.22402/j.rdipycs. unam.4.2.2018.183.195-213

Von Dawans, B., Kirschbaum, C., \& Heinrichs, M. (2011). The Trier Social Stress Test for Groups (TSST-G): A new research tool for controlled simultaneous social stress exposure in a group format. Psychoneuroendocrinology, 36(4), 514-522. doi: 10.1016/j.psyneuen.2010.08.004 


\section{Meta-Análisis del Artículo}

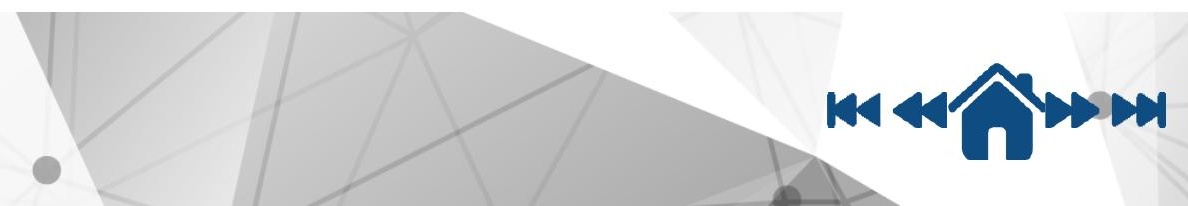


Revista Digital Internacional de Psicología y Ciencia Social |Vol. $6 \mid$ Núm. $2 \mid$ Julio-Diciembre 2020 | e-ISSS 2448-8119

\section{Dimensión Cuantitativa}

\section{Perfil de Evaluación entre pares}
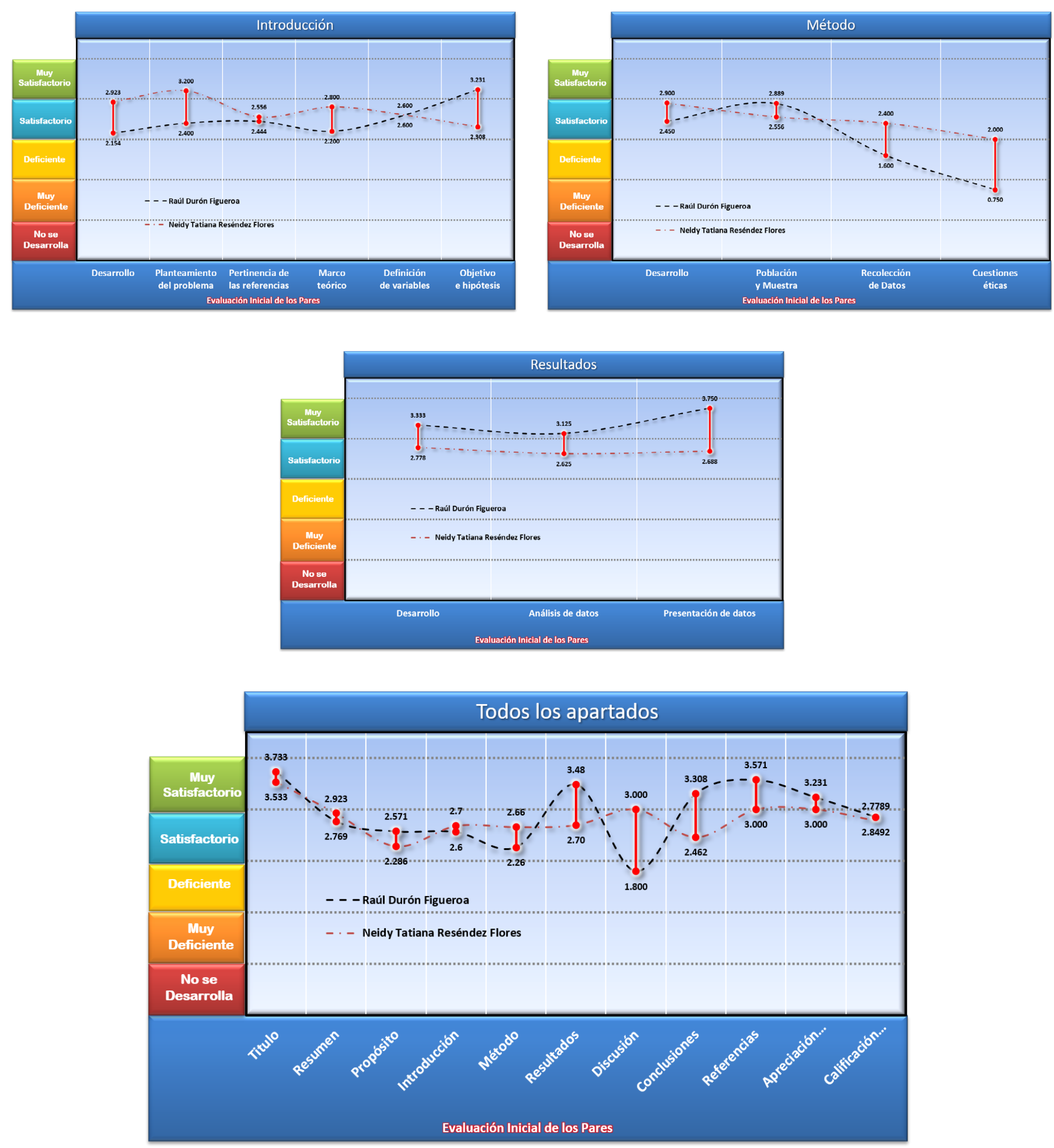


\section{Índice de Concordancia}

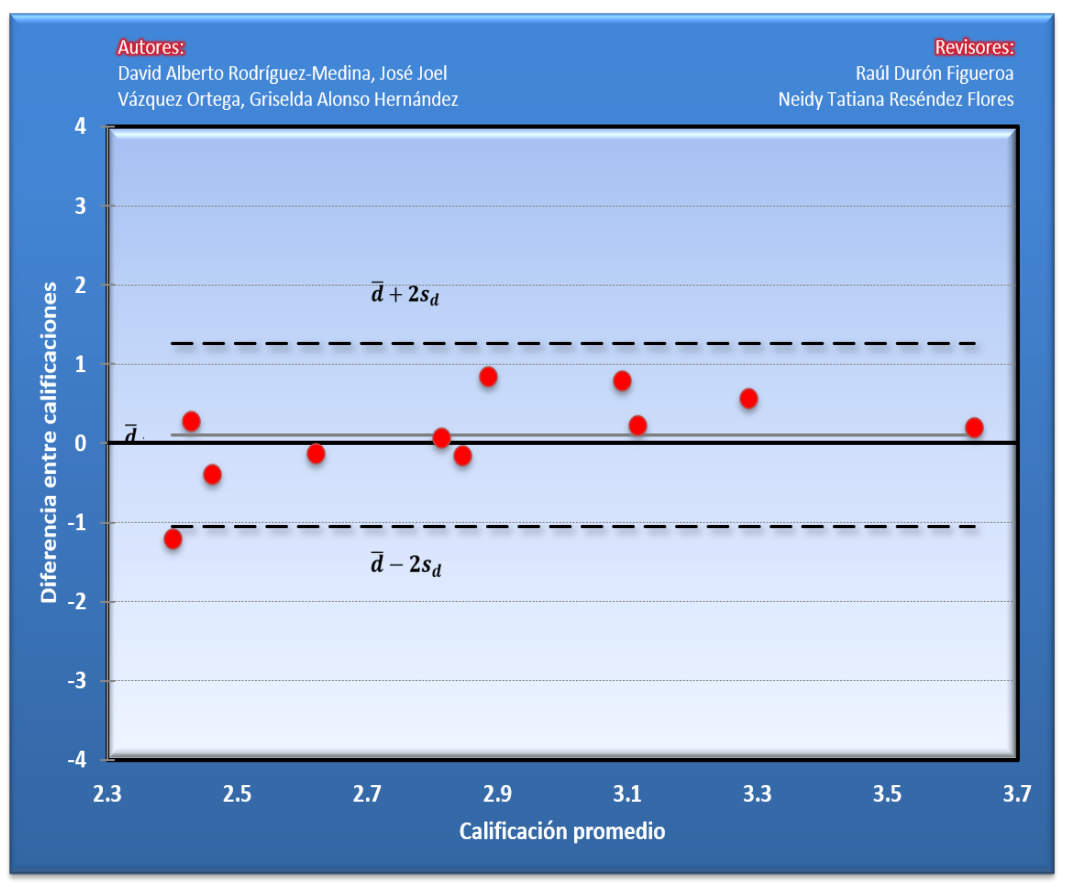

Índice de Acuerdo

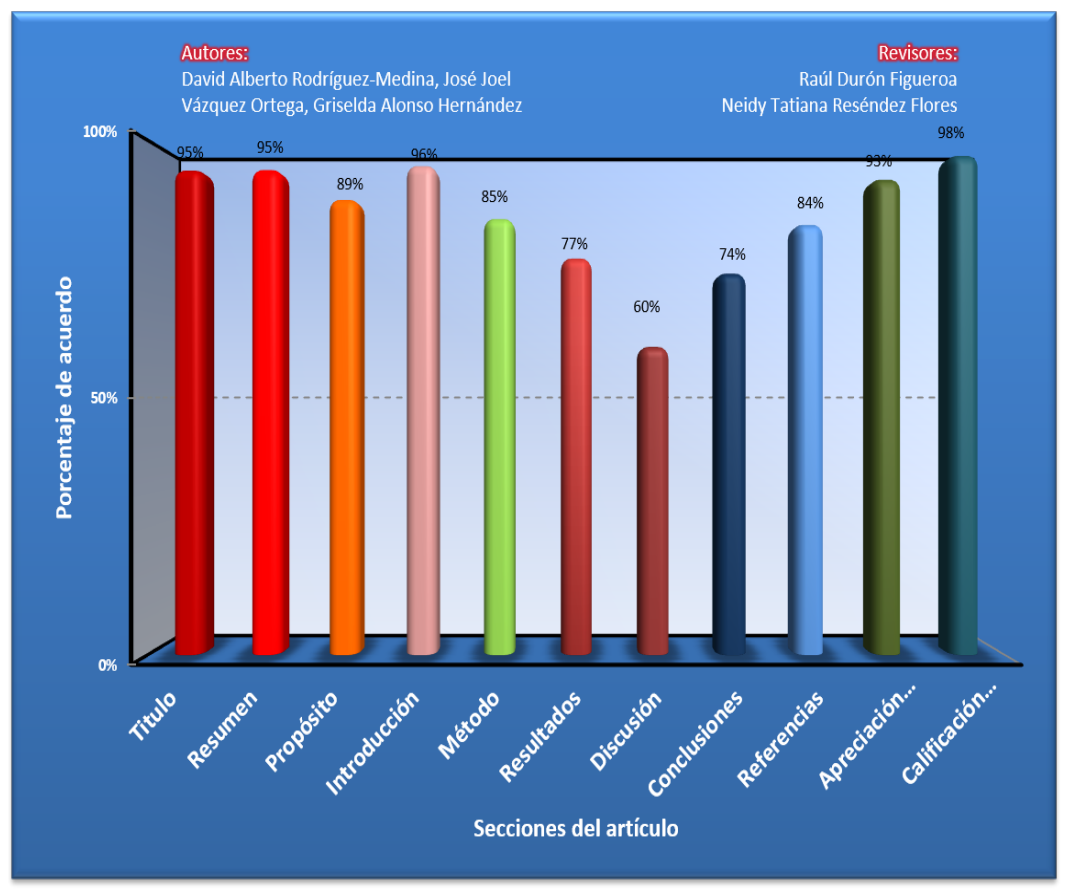




\begin{tabular}{|c|c|}
\hline Revisor 1 & Revisor 2 \\
\hline Raúl Durón Figueroa & Neidy Tatiana Reséndez Flores \\
\hline \multicolumn{2}{|c|}{ Título/Autoría } \\
\hline En general es un título pertinente al estudio. & Sin sugerencias \\
\hline \multicolumn{2}{|c|}{ Resumen } \\
\hline Excede el número de palabras propuesto & $\begin{array}{l}\text { Se deben seguir las directrices por párrafos y la infor- } \\
\text { mación debe estar de acuerdo al cuerpo del estudio. * } \\
\text { Observaciones en archivo anexo. }\end{array}$ \\
\hline \multicolumn{2}{|c|}{ Próposito del Estudio } \\
\hline $\begin{array}{l}\text { No queda claro la relación entre el estrés social y la me- } \\
\text { todología empleada }\end{array}$ & $\begin{array}{l}\text { Se deben especificar mejor el objetivo general, y objeti- } \\
\text { vos específicos. Estos deben tener relación con el proce- } \\
\text { dimiento y análisis estadísticos. Se debe complementar } \\
\text { información en este apartado: Tomar en cuenta cada } \\
\text { variable para la información que se anexa. Especificar } \\
\text { mejor la importancia del problema. }\end{array}$ \\
\hline \multicolumn{2}{|c|}{ Introducción } \\
\hline $\begin{array}{l}\text { Se presenta una introducción muy breve donde se reco- } \\
\text { mienda ampliar la revisión de la literatura y la descrip- } \\
\text { ción de las variables de estudio. }\end{array}$ & $\begin{array}{l}\text { Se deben especificar mejor los objetivos del estudio, no } \\
\text { queda claro cuál es el objetivo general y cuáles son los } \\
\text { específicos. }\end{array}$ \\
\hline \multicolumn{2}{|c|}{ Método } \\
\hline $\begin{array}{l}\text { No menciona las propiedades psicométricas de los ins- } \\
\text { trumentos y si estos están validados en la población } \\
\text { meta. Descripción deficiente del protocolo de estrés } \\
\text { social que se menciona. No describe el reclutamiento. }\end{array}$ & $\begin{array}{l}\text { Incluir datos sociodemográficos como sexo de los partici- } \\
\text { pantes, edad, semestre cursado, para mejorar información. } \\
\text { Mencionar los criterios de inclusión. El tamaño deseado } \\
\text { de la muestra, y el tamaño final. Detallar el procedimiento } \\
\text { de selección de participantes. Los análisis estadísticos se } \\
\text { detallan de acuerdo a los objetivos específicos para al final } \\
\text { llegar al objetivo general, es por eso que se deben especi- } \\
\text { ficar mejor los objetivos en el apartado de Procedimiento. }\end{array}$ \\
\hline
\end{tabular}




\begin{tabular}{|c|c|}
\hline Revisor 1 & Revisor 2 \\
\hline \multicolumn{2}{|c|}{ Resultados } \\
\hline Sin observaciones. & $\begin{array}{l}\text { Anexar los resultados de la selección de participantes y } \\
\text { datos sociodemográficos. Especificar mejor la informa- } \\
\text { ción en Tabla } 1 \text { para mejor entendimiento. Mencionar } \\
\text { el Flujo de participantes y la Selección de los sujetos } \\
\text { para el análisis de datos de acuerdo a las Directrices } \\
\text { para autores de este apartado. }\end{array}$ \\
\hline \multicolumn{2}{|c|}{ Discusión } \\
\hline $\begin{array}{l}\text { No se incluyen trabajos antecedentes para la interpre- } \\
\text { tación con los resultados obtenidos. No se detallan im- } \\
\text { plicaciones teóricas. }\end{array}$ & $\begin{array}{l}\text { Mejorar discusión sobre cada uno de los resultados ob- } \\
\text { tenidos de acuerdo a las variables de estudio. }\end{array}$ \\
\hline \multicolumn{2}{|c|}{ Conclusiones } \\
\hline Sin comentarios. & $\begin{array}{l}\text { Mejorar conclusión de acuerdo a las directrices para au- } \\
\text { tores: ¿Cuál es la importancia teórica, clínica o práctica } \\
\text { de los resultados y cuál la base para estas interpretacio- } \\
\text { nes? Si los descubrimientos son válidos y replicables. } \\
\text { ¿Qué fenómenos reales pueden ser explicados o mode- } \\
\text { lados por los resultados? ¿La investigación garantiza las } \\
\text { aplicaciones? ¿Qué problemas siguen sin resolverse o } \\
\text { cuáles surgen a causa de estos descubrimientos? }\end{array}$ \\
\hline \multicolumn{2}{|c|}{ Referencias } \\
\hline Sin observaciones. & Sin sugerencias \\
\hline
\end{tabular}




\section{Historia del Proceso}

\section{EDITORIAL}

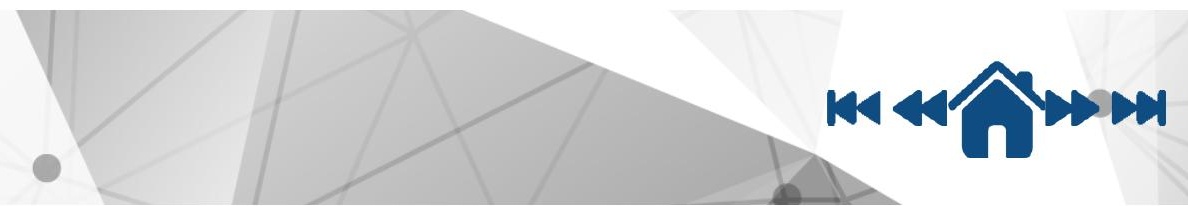

